\title{
An Advanced Fitting Method for Crystallographic and Morphological Analyses of EBSD Data Applied for Low-Carbon Steel Martensite
}

\author{
Anh H. Pham*1,*2, Takuya Ohba*2, Shigekazu Morito and Taisuke Hayashi \\ Department of Materials Science, Shimane University, Shimane 690-8504, Japan
}

Some essential modifications were introduced to the ordinary fitting method to make it become a powerful tool for thorough crystallographic and morphological analyses of lath martensite based on electron backscatter diffraction (EBSD) data. A large area of martensite which covers a whole prior austenite grain can be effectively analyzed. The austenite orientation and orientation relationship between martensite and austenite can be obtained by fitting from martensite orientations. The fitting procedure is fast with high precision up to 0.5 degrees for austenite orientation and 0.2 degrees for the orientation relationship. The output data are also modified for automatically indexing martensite variants and visual plotting of the results. [doi:10.2320/matertrans.M2012355]

(Received October 30, 2012; Accepted May 14, 2013; Published June 21, 2013)

Keywords: lath martensite, fitting method, electron backscatter diffraction (EBSD), austenite orientation, orientation relationship

\section{Introduction}

Recently, EBSD method has been used intensively to study morphology and crystallography of martensite in steel. The EBSD method allows a large area of martensite up to $1 \mathrm{~mm}^{2}$ to be analyzed. Many aspects of morphology can be obtained such as, packet size, block and sub-block sizes, and their distribution maps. For analyzing martensite structures, essential crystallographic information is orientation relationship between parent austenite and transformed martensite. If transformed martensite contains enough amount of retained austenite, one can easily deduce the orientation relationship from EBSD data. However, one cannot readily obtain orientation relationship for martensite with little amount of retained austenite, such as martensite of low-carbon steels. In previously reported papers the orientation relationship is often supposed to be KurdjumovSachs (K-S) type, ${ }^{1-3)}$ though the results shown a certain deviation from the exact K-S. ${ }^{2,3)}$ A numerical fitting method had been applied for orientation relationship analysis of martensite and bainite in steels by Miyamoto et al. ${ }^{4)}$ with dual fitting of both austenite orientation and orientation relationship by a single fitting criterion. The obtained orientation relationship is reported to be close to GreningerTroiano $(\mathrm{G}-\mathrm{T})^{5)}$ type and locally varied within one prior austenite grain due to transformation strain. ${ }^{4)}$ Until now, the main disadvantage of fitting method to obtain orientation relationship from observed martensite orientation is that the process takes a lot of time for finding a solution from a relatively small EBSD data set (thousands of scan points). If taking calculation time into consideration, the method is only capable to obtain orientation relationship from a part of austenite grain, instead of the whole grain. When small parts of one prior austenite are used for fitting austenite orientation and orientation relationship, the scatter of obtained results can increase up to $\pm 1^{\circ}$, especially for austenite orientation. By introducing some essential modifications of calculation

\footnotetext{
${ }^{* 1} \mathrm{Ph}$.D. Student, Shimane University

${ }^{* 2}$ Corresponding author, E-mail: s119706@matsu.shimane-u.ac.jp, ohba@ riko.shimane-u.ac.jp
}

and fitting, we are now able to do a fitting procedure for a large EBSD set (hundred thousands of scan points), which covers a whole prior austenite grain for a relatively short running time. Thus the precision of fitting method is improved with a reliable and reproducible result. Moreover, by using the fitting method, we provide other advantages which allow automatically indexing martensite variants and visually plotting of various analytical results.

\section{Calculation Method}

Generally the orientation of martensite variants can be generated from given austenite orientation and orientation relationship between austenite and martensite by eq. (1). All of the orientations mentioned herein are in form of $3 \times 3$ matrices.

$$
M_{i_{\text {standard }}}^{\mathrm{bcc}}=\left(O R_{1}^{\mathrm{fcc}-\mathrm{bcc}} C_{i}\right) A^{\mathrm{fcc}}, \quad i=1-24 .
$$

Where, $M_{i_{\text {standard }}}^{\mathrm{bcc}}$ : orientation of $i^{\text {th }}$ standard martensite variants generated by given austenite orientation and orientation relationship; $A^{\text {fcc }}$ : orientation of austenite; $C_{i}: i^{\text {th }}$ conversion matrix of 24 symmetry operations for cubic system (Table 1); $O R_{1}^{\mathrm{fcc}-\mathrm{bcc}}$ : the 1st variant of orientation relationship in martensite frame.

To find a solution for austenite orientation and orientation relationship from EBSD data of experimental martensite orientations, one should find a standard martensite variant $M_{i_{\text {standard }}}^{\mathrm{bcc}}$ which has the smallest misorientation $\Delta \theta_{k}$ from a given $k^{\text {th }}$ experimental orientation $M_{k_{\text {experiment }}}^{\text {bcc }}$ (eq. (2))

$$
\begin{aligned}
\Delta \theta_{k} & =\min \left\{\arccos \left(\left[\operatorname{tr}\left(M_{i_{\text {standard }}}^{\mathrm{bcc}}\left(M_{k_{\text {experiment }}}^{\mathrm{bcc}} C_{j}\right)^{-1}\right)-1\right] / 2\right)\right\}, \\
\forall i, j & =1-24 .
\end{aligned}
$$

Where, $\operatorname{tr}\left(M_{i_{\text {standard }}}^{\mathrm{bcc}}\left(M_{k_{\text {experiment }}}^{\mathrm{bcc}} C_{j}\right)^{-1}\right)$ is the trace of misorientation matrix $M_{i_{\text {standard }}}^{\text {bcc }}\left(M_{k_{\text {experiment }}}^{\text {bcc }} C_{j}\right)^{-1}$. The solution can be found by minimization of average misorientation over all experimental data $\Delta \theta_{\text {average }}=\sum_{k=1}^{N} \Delta \theta_{k} / N \rightarrow \min$, where $N$ is the number of scan points.

Due to the symmetry, an arbitrary orientation in cubic system can be represented by any of 24 mathematically distinguished matrices. Hence, the total number of matrix operations, which is required to find the minimum miso- 
Table 1 Twenty four conversion matrices for cubic system.

$$
\begin{gathered}
C_{1}=\left[\begin{array}{lll}
1 & 0 & 0 \\
0 & 1 & 0 \\
0 & 0 & 1
\end{array}\right] \\
C_{7}=\left[\begin{array}{lll}
0 & 0 & 1 \\
0 & \overline{1} & 0 \\
1 & 0 & 0
\end{array}\right] \\
C_{13}=\left[\begin{array}{lll}
0 & 1 & 0 \\
1 & 0 & 0 \\
0 & 0 & 1
\end{array}\right] \\
C_{19}=\left[\begin{array}{lll}
1 & 0 & 0 \\
0 & 0 & 1 \\
0 & 1 & 0
\end{array}\right]
\end{gathered}
$$

$$
\begin{aligned}
& C_{3}=\left[\begin{array}{lll}
0 & 0 & 1 \\
1 & 0 & 0 \\
0 & 1 & 0
\end{array}\right] \\
& C_{9}=\left[\begin{array}{lll}
1 & 0 & 0 \\
0 & 0 & 1 \\
0 & 1 & 0
\end{array}\right] \\
& C_{15}=\left[\begin{array}{lll}
0 & 0 & 1 \\
0 & 1 & 0 \\
\overline{1} & 0 & 0
\end{array}\right] \\
& C_{21}=\left[\begin{array}{lll}
0 & 1 & 0 \\
1 & 0 & 0 \\
0 & 0 & 1
\end{array}\right]
\end{aligned}
$$

$$
\begin{aligned}
C_{4} & =\left[\begin{array}{lll}
0 & \overline{1} & 0 \\
\overline{1} & 0 & 0 \\
0 & 0 & \overline{1}
\end{array}\right] \\
C_{10} & =\left[\begin{array}{lll}
0 & 1 & 0 \\
0 & 0 & \overline{1} \\
\overline{1} & 0 & 0
\end{array}\right] \\
C_{16} & =\left[\begin{array}{lll}
1 & 0 & 0 \\
0 & \overline{1} & 0 \\
0 & 0 & \overline{1}
\end{array}\right] \\
C_{22} & =\left[\begin{array}{lll}
0 & 0 & 1 \\
\overline{1} & 0 & 0 \\
0 & \overline{1} & 0
\end{array}\right]
\end{aligned}
$$

$$
\begin{aligned}
C_{6} & =\left[\begin{array}{lll}
\overline{1} & 0 & 0 \\
0 & 0 & \overline{1} \\
0 & \overline{1} & 0
\end{array}\right] \\
C_{12} & =\left[\begin{array}{lll}
0 & 0 & \overline{1} \\
\overline{1} & 0 & 0 \\
0 & 1 & 0
\end{array}\right] \\
C_{18} & =\left[\begin{array}{lll}
0 & \overline{1} & 0 \\
0 & 0 & \overline{1} \\
1 & 0 & 0
\end{array}\right] \\
C_{24} & =\left[\begin{array}{lll}
\overline{1} & 0 & 0 \\
0 & \overline{1} & 0 \\
0 & 0 & 1
\end{array}\right]
\end{aligned}
$$

rientation angle $\Delta \theta_{k}$ for a given $k^{\text {th }}$ experimental orientation $M_{k_{\text {experiment }}}^{\text {bcc }}$ in eq. (2), is $24 \times 24=576$ operations.

Each orientation of cubic system in a three dimensional reference frame is characterized by three independent variables as minimum. To find both austenite orientation and orientation relationship simultaneously we have to deal with six independent variables $x_{i}, i=1-6$. The numerical fitting approach was applied for finding the global minimum of $\Delta \theta_{\text {average }}$ with a given fitting interval $d$ and fitting step $s$. All six variables are allowed to change independently from $x_{i}^{o}-d$ to $x_{i}^{o}+d$ with increasing step $s$, where $x_{i}^{o}$ are the initially given values of $x_{i}$. Obviously, the global minimum of $\Delta \theta_{\text {average }}$ within fitting interval $\pm d$ can be found with the error proportional to the fitting step $s$. By this algorithm, the total matrix operations which are required to find both austenite orientation and OR by using 6 variables are increased by $f^{3} \times f^{3}=f^{6}$ times, where $f=2 d / s$, is the number of fitting steps.

Generally the total number of matrix operation which required for fitting both austenite orientation and orientation relationship by the conventional fitting method for EBSD data set with $N$ scan points is

$$
f^{3} \times f^{3} \times 24 \times 24 \times N=576 f^{6} N .
$$

For a normal data set of 30,000 scan points with number of fitting steps $f=10$, the total number of matrix operation is $1.72 \times 10^{13}$. The large required number of matrix operation explains why the fitting method for a normal or large EBSD data set takes a lot of operating time. If one would like to have the result for a reasonable calculation time, the available data set should be quite small as several thousand of scan points. For a normal EBSD mode with $0.2 \mu \mathrm{m}$ scanning step, at least 62,500 scan points are required to observe a whole prior austenite grain, which size is normally more than $2,500 \mu \mathrm{m}^{2}$ for low-carbon steel. In this paper, we applied modifications to the conventional fitting method to improve its performance and reliability as described below.

\subsection{Fitting austenite orientation and orientation rela- tionship separately}

The orientation relationship between lath martensite and austenite is reported to be varying from K-S, NishiyamaWassermann $(\mathrm{N}-\mathrm{W})$ or G-T by different researchers working on different sample steels. ${ }^{1-8)}$ However, all the reported orientation relationships are quite close to each other. For instant, the difference between K-S and N-W is only $5.26^{\circ}$. The misorientation between $\mathrm{K}-\mathrm{S}$ and G-T is $2.40^{\circ}$, while misorientation between $\mathrm{N}-\mathrm{W}$ and G-T is $2.86^{\circ}$. A TEM observation by Kelly et l. $^{7)}$ showed that the orientation relationship in case of lath martensite is almost constant although the orientation relationships were extracted from very thin and heavily deformed retained austenite films and neighbor martensite regions. Based on this fact, we consider that the orientation relationship is constant or almost constant for given steel with a known chemical composition.

Using this idea, we have been tried to fix the orientation relationship for fitting only austenite orientation to reduce the number of calculation operations. For the first trial we used K-S orientation relationship to fit austenite orientation. Note that the austenite orientation term used here means average orientation overall the prior austenite grain before martensitic transformation. After finding a solution of austenite orientation, the orientation relationship was fitted by fixing austenite orientation. The new fitted orientation relationship is found to be close to G-T for various steels with different chemical composition. For further fitting, the obtained orientation relationship will be used as the initial orientation relationship instead of K-S to fit austenite orientation, since the initial orientation relationship is closer to the fitted orientation relationship than the K-S. By using this approach the multiple to the operations which are required to find both austenite orientation and orientation relationship by numerical fitting is equal to $2 \times f^{3}$ instead of $f^{6}$.

\subsection{Using the characteristics of (001) pole figure}

The (001) pole figure of experimental points (Fig. 1) for typical EBSD data of martensite within one prior austenite grain shows a ring-like distribution of $(001)_{\alpha^{\prime}}$ martensite orientations around each pole of $(001)_{\gamma}$ austenite orientation, which is referred as a Bain group. Within a Bain group, there are 8 martensite variants and the misorientations between $(001)_{\alpha^{\prime}}$ poles of 8 martensite variants and the corresponding $(001)_{\gamma}$ austenite pole are ideally the same and smaller than $15^{\circ}$ for all reported orientation relationships. By classifying the experimental points of martensite into three Bain groups, we now have to deal with only 8 martensite variants in a Bain group instead of all 24 variants. Furthermore, the operation, which we have to deal with, is only between two vectors. 


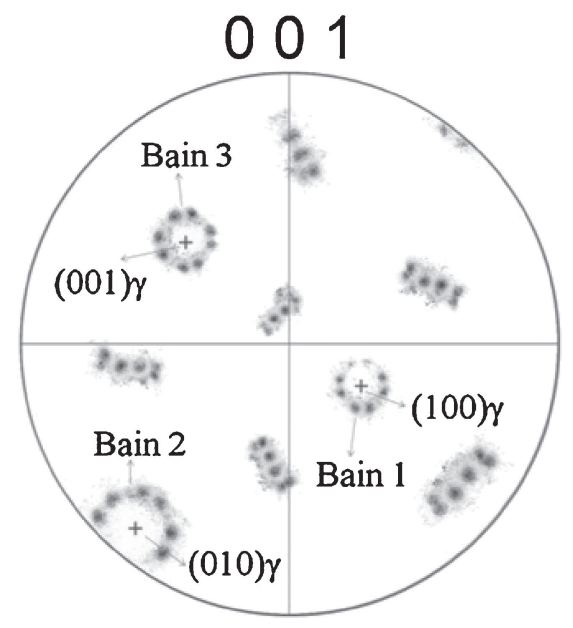

Fig. 1 Typical (001) pole figure of lath martensite transformed from one prior austenite grain.

Assuming a martensite orientation is expressed in a matrix form as $\left(m_{i}\right), i=1-3$, where $m_{i}$ are three orthogonal unit vectors. In the same manner, the orientation of austenite is $\left(a_{j}\right), j=1-3$. As seen in the Fig. 1, the martensite orientation belongs to a given Bain group $k(k=1-3)$ if and only if the minimum angle between $m_{i}$ and $a_{j}$ is equal to the minimum angle between $m_{i}$ and $a_{k}$

$$
\min \left\{\angle\left(m_{i}, a_{j}\right)\right\}=\min \left\{L\left(m_{i}, a_{k}\right)\right\}, \quad \forall i, j=1-3 .
$$

The total vector operations required to find such of vector $a_{k}$ for a given martensite orientation $\left(m_{i}\right)$, is $3 \times 3=9$. Hence, it requires 9 vector operations to find the closest Bain group and 8 vector operations to find the candidates for the closest martensite variant in the Bain group. The total vector operations required for finding the closest martensite variant to a given experimental point are $9+8=17$ operations. If we consider three vector operations are equal to one matrix operation, the number of equivalent matrix operations is only 6 for each experimental point.

\subsection{Simplifying the calculation of misorientation}

From pole figure we have already noted that the misorientation between the experimental point and the nearest standard martensite variant is small and less than $15^{\circ}$. We also made an assumption that, if there is a combination of two cubic orientations $\mathrm{O} 1(\mathrm{Oxyz})$ and $\mathrm{O} 2$ $\left(\mathrm{O} x^{\prime} y^{\prime} z^{\prime}\right)$ with small misorientation providing that $\alpha_{x}=$ $\min \left\{\alpha_{x}, \beta_{x}, \gamma_{x}\right\}$, where $\alpha_{x}=\angle\left(\mathrm{O} x, \mathrm{O} x^{\prime}\right), \beta_{x}=\angle\left(\mathrm{O} y, \mathrm{O} x^{\prime}\right)$, $\gamma_{x}=\angle\left(\mathrm{O} z, \mathrm{O} x^{\prime}\right) ;$ by analogy $\alpha_{y}=\min \left\{\alpha_{y}, \beta_{y}, \gamma_{y}\right\}, \alpha_{z}=$ $\min \left\{\alpha_{z}, \beta_{z}, \gamma_{z}\right\}$, then the misorientation calculated for the given combination is the smallest and can be accepted as the true misorientation between $\mathrm{O} 1$ and $\mathrm{O} 2$. Now we will approve this assumption. When the orientation $\mathrm{O} 1$ is fixed as a reference coordinate system $\mathrm{Oxyz}$, the trace of rotation matrix from $\mathrm{O} 2$ to $\mathrm{O} 1$ will be equal to $\cos \left(\alpha_{x}\right)+\cos \left(\alpha_{y}\right)+$ $\cos \left(\alpha_{z}\right)$. The misorientation angle $\theta$ between $\mathrm{O} 1$ and $\mathrm{O} 2$ is

$$
\cos \theta=\left(\cos \alpha_{x}+\cos \alpha_{y}+\cos \alpha_{z}-1\right) / 2 .
$$

If misorientation between $\mathrm{O} 1$ and $\mathrm{O} 2$ is known to be smaller than $15^{\circ}$ the minimum misorientation (Fig. 2) can be deduced from the eq. (5), providing that

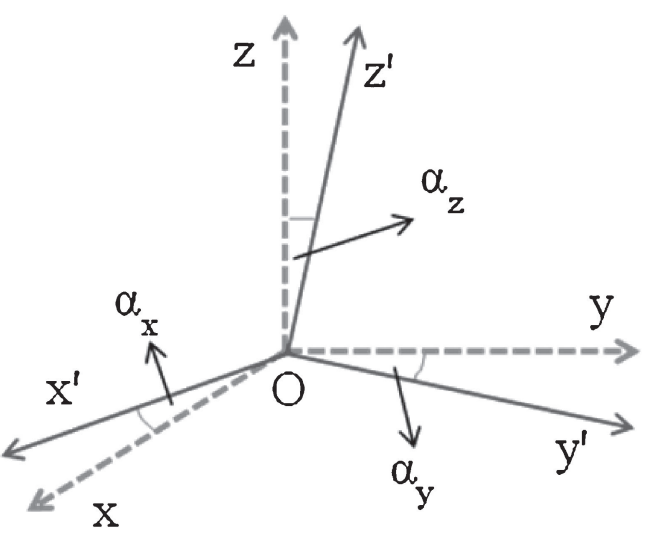

Fig. 2 A symmetry of orientation $\mathrm{O} 2\left(\mathrm{O} x^{\prime} y^{\prime} z^{\prime}\right)$ in frame of orientation $\mathrm{O} 1$ (Oxyz) giving the smallest misorientation between them.

$$
\begin{aligned}
& \alpha_{x}=\min \left\{\alpha_{x}, \beta_{x}, \gamma_{x}\right\}, \quad \alpha_{y}=\min \left\{\alpha_{y}, \beta_{y}, \gamma_{y}\right\}, \\
& \alpha_{z}=\min \left\{\alpha_{z}, \beta_{z}, \gamma_{z}\right\} .
\end{aligned}
$$

Since $\left(\cos \alpha_{x}\right)^{2}+\left(\cos \beta_{x}\right)^{2}+\left(\cos \gamma_{x}\right)^{2}=1$ then $\cos \alpha_{x} \geq$ $1 / \sqrt{3}$ or $\alpha_{x}<\arccos (1 / \sqrt{3}) \approx 55^{\circ}$, by analogy $\alpha_{y}$ and $\alpha_{z}<55^{\circ}$. Thus, $\cos \theta$ calculated from eq. (5) will have maximum value or $\theta$ will be minimum misorientation angle.

By using this theorem we avoided using 24 conversion matrices $C_{i}$ for finding the misorientation between $\mathrm{O} 1$ and $\mathrm{O} 2$. Therefore we can use only one matrix operation instead of twenty four to find the true misorientation between the two close orientations.

In addition to the above mentioned modifications (in 2.1 and 2.2), the total number of matrix operations required for fitting austenite orientation and orientation relationship is

$$
2 \times f^{3} \times 6 \times N=12 f^{3} N .
$$

For the same value of $N$ and $f$ given above, the total number of required matrix operations is $3.6 \times 10^{8}$. With the modifications described in section 2.1-2.3, we were able to reduce the required matrix operations by $4.8 \times 10^{4}$ times.

\subsection{Using two criteria for fitting}

When using minimum average misorientation as the only criterion for fitting, we often found an erroneous solution of austenite orientation. It is found that the erroneous solution well minimizes the average misorientation, but only a small number of experimental points are assigned to calculated martensite variants within a given tolerance of misorientation. Hence, we introduced an additional criterion that the number of experimental points assigned to martensite variants within a given tolerance angle should be maximum.

Thus the two criteria for fitting are:

(1) minimum average misorientation $-\Delta \theta_{\text {average; }}$;

(2) maximum number of experimental points which can be assigned to martensite variants within a given tolerance angle- $N_{\text {fit }}$.

Ideally, the solution of austenite orientation may not simultaneously satisfy the smallest value of average misorientation $\Delta \theta_{\text {average }}$ and maximum number of fitted experimental points $-N_{\text {fit }}$. For numerical fitting, a combined criterion was made by dividing the average misorientation by the total number of fitted experimental points: 
$\Delta \theta_{\text {average }} / N_{\text {fit }}$. By using the combined criterion for the fitting, the solution now is reliable and reproducible during a repeated running cycle with any set of EBSD data.

\subsection{Modifying the data for automatically visual plotting of results}

One who studies morphology of lath martensite often has problem with plotting the characteristic features of morphology such as packet, block, or variant (sub-block) maps, since these kinds of plots require manual indexing of martensite variants. An important advantage of this calculation is that after fitting austenite orientation and orientation relationship, we are able to analyze the data and modify the output file for automatically indexing martensite variants. After the treatment, various kinds of visual plotting can be plotted automatically and easily on the common commercial program for EBSD data analysis. Some examples of crystallographic and morphological analyses will be given for illustration and discussion.

\section{Experimental}

A low-carbon 0.1 mass $\% \mathrm{C}-3$ mass $\% \mathrm{Mn}$ steel was investigated. The thin specimen was heated to $1327 \mathrm{~K}$ by electric resistance with $150 \mathrm{~s}$ holding time, followed by a rapid quenching under high pressure water-jet. This heat treatment was aimed to obtain a microstructure of full lath martensite with average prior austenite grain size of $70 \mu \mathrm{m}$. The sample was cut for EBSD observation from a center part of the specimen and mechanically polished. The EBSD experiment was conducted on a JEOL JSM7001FA field emission gun scanning electron microscope equipped with TSLCEBSD data collection system. The scan was performed at $25 \mathrm{kV}$ accelerating voltage and $13 \mathrm{nA}$ probe current with scanning step of $0.2 \mu \mathrm{m}$ at $\times 1000$ magnification. Three prior austenite grains which were numbered as grain 1, grain 2 and grain 3, were analyzed by the improved fitting program. Five different parts of the grain 1 (see Fig. 3), which are notated from P1 to P5, were also used for fitting austenite orientation and orientation relationship (Table 3).

\section{Results and Discussion}

\subsection{Coarse fitting and fine fitting}

Based on the above described algorithm, a computer program for fitting austenite orientation and OR was written in Fortran 90, which can run on any personal computer (PC) with Windows operating system. For certain demands, the fitting procedure can be processed either in coarse mode or in fine mode. A coarse fitting mode includes only coarse fitting phase, while a fine fitting mode requires both coarse and fine fitting phases. The coarse fitting phase uses the trial austenite orientation and the general orientation relationship as input parameters. The fitting interval is from -3 to $+3^{\circ}$ and the fitting step is $1^{\circ}(f=6)$. Fine fitting phase uses the results of coarse fitting phase as the input. Fitting interval is from -1 to $+1^{\circ}$ and the fitting step is $0.2^{\circ}(f=10)$. Figure 4 shows how the running time of coarse and fine fitting mode for finding both austenite orientation and orientation relationship depend on the data volume. The EBSD data of the grain 1, which has

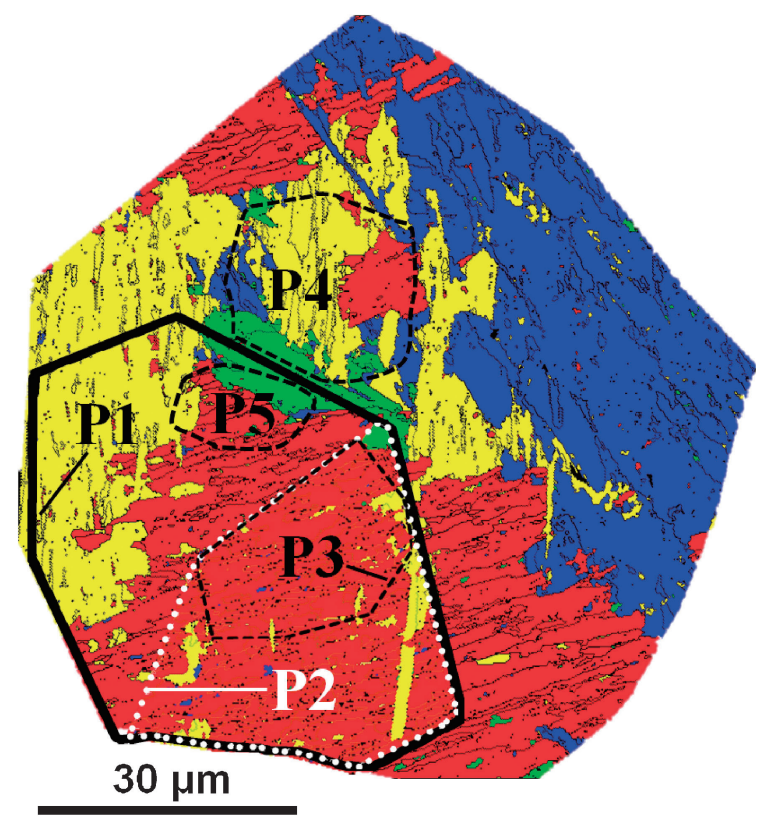

Fig. 3 Grain 1 with its five parts (from P1 to P5) used for fitting austenite orientation and orientation relationship, the martensite variants are colored following parallel relationship between $(011)_{\alpha^{\prime}}$ and four variants of $(111)_{\gamma}$ close-packed plane. The contour of P1 is denoted by solid black line, contour of P2 by dotted white line and contours of P3-P5 by dashed black line.

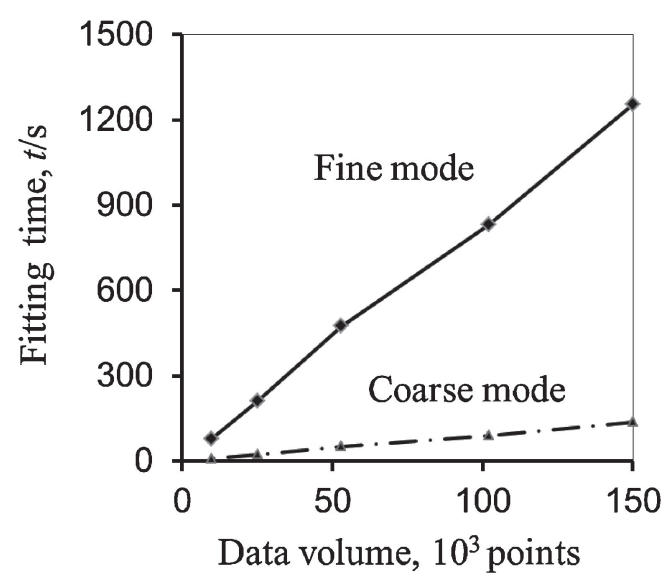

Fig. 4 Fitting time for both austenite orientation and OR versus volume of data set.

153,000 scan points, was analyzed in both coarse and fine fitting mode. The difference between the obtained austenite orientations is $0.33^{\circ}$, while the orientation relationships are identical. The coarse mode can be applied for rapid fitting austenite orientation and orientation relationship since it is much faster than fine fitting with precision better than $0.5^{\circ}$. For presenting the best capability of the improved method, all further examples are calculated by using the fine mode. The calculation speed was estimated on a normal Windows PC equipped with an Intel ${ }^{\circledR}$ Core $^{\mathrm{TM}} \mathrm{i} 5-3.33 \mathrm{GHz}$ processor and $4 \mathrm{~GB}$ of memory.

\subsection{Effect of scanning step on fitting results}

For grain 1 (Fig. 3), the EBSD data set with original scanning step $0.2 \mu \mathrm{m}$ was coarsened by using TSL OIM 
Table 2 Effect of coarsening EBSD data of grain 1 on fitting results.

\begin{tabular}{|c|c|c|c|c|c|c|}
\hline Number of coarsening cycles & & 0 & 1 & 2 & 3 & 4 \\
\hline Scanning step, $\mu \mathrm{m}$ & & 0.2 & 0.4 & 0.8 & 1.6 & 3.2 \\
\hline Data volume, point & & 153,252 & 38,342 & 9,592 & 2,413 & 608 \\
\hline Calculation time, $t / \mathrm{s}$ & & 1287 & 351 & 103 & 21 & 6 \\
\hline \multirow{2}{*}{ Deviation from fitting of original data, $\theta /{ }^{\circ}$} & Austenite orientation & 0.00 & 0.00 & 0.00 & 0.00 & 0.14 \\
\hline & Orientation relationship & 0.00 & 0.07 & 0.07 & 0.09 & 0.15 \\
\hline
\end{tabular}

Table 3 Details of EBSD data used for austenite orientation and orientation relationship fitting.

\begin{tabular}{|c|c|c|c|c|c|c|c|c|}
\hline Name & Grain 1 & Part 1 & Part 2 & Part 3 & Part 4 & Part 5 & Grain 2 & Grain 3 \\
\hline Notation & G1 & $\mathrm{P} 1$ & $\mathrm{P} 2$ & P3 & P4 & P5 & G2 & G3 \\
\hline Data volume, point & 153,000 & 53,694 & 25,557 & 9,885 & 9,820 & 3,638 & 167,043 & 152,352 \\
\hline $\begin{array}{l}\text { Number of main martensite variants } \\
\text { with fraction larger than } 0.01\end{array}$ & 18 & 10 & 8 & 6 & 17 & 8 & 18 & 17 \\
\hline
\end{tabular}

program which artificially doubled the scanning step per one coarsening cycle. After each coarsening cycle the data volume is decreased by 4 times, so the calculation time is reduced by about 4 times. The details of coarsened data are given in Table 2. Up to three coarsening cycles the obtained results of austenite orientation for original data and coarsened data are ideally the same, whereas the tolerance of obtained orientation relationships is $0.1^{\circ}$ (Table 2). It shows that using coarse data with scanning step up to $1.6 \mu \mathrm{m}$ is an effective way to reduce calculation time without the risk of losing the precision. By this measure, a large number of prior austenite grains or a very large grain can be effectively analyzed within reasonable time.

\subsection{Fitting for different parts of one austenite grain and for different austenite grains}

EBSD data of five different parts of the grain 1, which contours are shown in Fig. 3, were also used for analyses. In this figure, martensite variants are colored by their plane parallel relationship with austenite, namely $(011)_{\alpha^{\prime}} / /(111)_{\gamma}$ or close packed plane variant 1 (CP1)-red, $(011)_{\alpha^{\prime}} / /(1 \overline{1} 1)_{\gamma}$ or CP2-yellow, $(011)_{\alpha^{\prime}} / /(\overline{1} 11)_{\gamma}$ or CP3-green and $(011)_{\alpha^{\prime}} / /$ $(11 \overline{1})_{\gamma}$ or CP4-blue. All the five parts are cropped from original grain 1 with $0.2 \mu \mathrm{m}$ scanning step. Part 1 is cut from grain 1 so that it contains mainly 10 martensite variants of $\mathrm{CP} 1, \mathrm{CP} 2$ and $\mathrm{CP} 3$. Also the data volume of part 1 is about 0.3 fractions of the grain 1 's. Part 2 which is a half of part 1 , contains 8 martensite variants of $\mathrm{CP} 1$ and $\mathrm{CP} 2$. Part 3 is cut from part 2 so that it covers 6 martensite variants of CP1 and CP2. Part 4 which has the same size as part 3, is cut from another region of grain 1 next to the part 1 . Despite its small size, part 4 contains 17 martensite variants of four $\mathrm{CP}$ variants. Part 5 is cut from a very small region of part 1 . This part covers 8 martensite variants of $\mathrm{CP} 1$ and $\mathrm{CP} 3$. The details of EBSD data of the five parts are given in Table 3 and Fig. 3.

The deviations of fitting results obtained for each part from the results obtained by fitting the whole grain 1 were given in Fig. 5. It shows that the deviation of fitted orientation

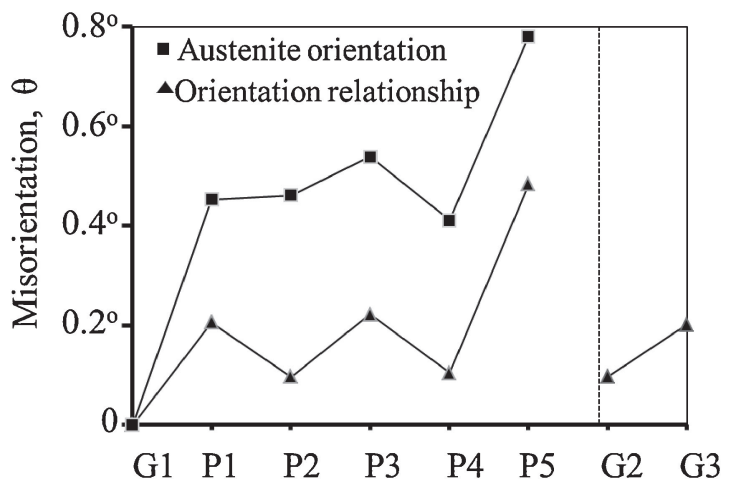

Fig. 5 Deviation of orientation relationships and austenite orientations obtained by fitting separately five parts of grain 1 as well as by fitting grain 2 and grain 3 from the results obtained by fitting grain 1 as a whole.

relationship for each part changes in correlation with its data volume and the number of included martensite variants. For the smallest part which contains only 3,638 scan points, the difference is $0.48^{\circ}$. The tolerance of fitting orientation relationship is $0.2^{\circ}$ for other parts of martensite which belong to the same prior austenite grain. The differences between orientation relationship obtained from fitting grain 1 and orientation relationships obtained from fitting grain 2 and grain 3 are within $0.2^{\circ}$.

The details of orientation relationship were presented by deviation between close-packed planes $\theta_{1}$ versus deviation between close-packed directions $\theta_{2}$ of austenite and martensite (Fig. 6). The mean orientation relationship is characterized by $\theta_{1}=1.72^{\circ}$ and $\theta_{2}=2.63^{\circ}$. This orientation relationship is close to G-T type, which has $\theta_{1}=1.0^{\circ}$ and $\theta_{2}=2.50^{\circ}$. Average deviation over all orientation relationships obtained by fitting the EBSD data provided in Table 3 from the mean value is only $0.12^{\circ}$. Thus, for given steel the orientation relationship between martensite and austenite can be confirmed to be almost constant, with the tolerance of $\pm 0.2^{\circ}$ for all austenite grains.

In a previous work $^{5)}$ for a 0.15 mass $\% \mathrm{C}-1.5$ mass $\% \mathrm{Mn}$ steel the orientation relationship between austenite and 
martensite $\left(15 \mathrm{C}^{\mathrm{M}}\right)$ was reported as $\theta_{1}=1.70^{\circ}$ and $\theta_{2}=3.0^{\circ}$, while the orientation relationship between austenite and bainite $\left(15 \mathrm{C}^{\mathrm{B}}\right.$ ) was $\theta_{1}=1.70^{\circ}$ and $\theta_{2}=2.6^{\circ}$ (Fig. 6). In comparison with our result the deviation between $\theta_{1}$ is very small, while the deviation between $\theta_{2}$ is about $0.4^{\circ}$ in case of austenite/martensite orientation relationship. Beside the reported fitting tolerance of about $\pm 0.3^{\circ}$, , $^{\text {) }}$ the deviation between orientation relationships obtained in previous and present works may be caused by the difference in chemical composition of studied steels. ${ }^{5)}$

Deviation between austenite orientations obtained by fitting from part 1 to part 4 and the result obtained by fitting whole the grain is within $0.5^{\circ}$. For part 5 the deviation is $0.78^{\circ}$ due to size effect of a very small part which contains only 3,638 scan points. It shows that a small part of one prior austenite grain, which covers about 0.1 fraction of the grain (as part 3 and 4) can be used for fitting austenite orientation giving the results with precision of $\pm 0.5^{\circ}$. Larger scatter of austenite orientation also imply that austenite orientations were locally changed due to transformation strain whereas the orientation relationship is almost unchanged.

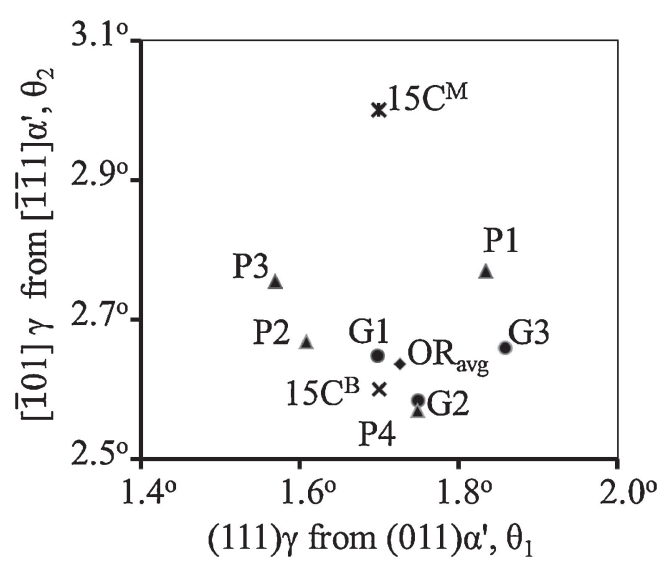

Fig. 6 The obtained orientation relationships plotted in term of deviation between close-packed planes versus deviation between close-packed directions of austenite and martensite. Notations here correspond to that of Fig. 3 and Table 3. See text for $15 \mathrm{C}^{\mathrm{B}}$ and $15 \mathrm{C}^{\mathrm{M}}$.

\subsection{Automatically indexing martensite variants and visual plotting of morphological features}

When the austenite orientation and orientation relationship between martensite and austenite are known, various crystallographic and morphological analyses will be available using only EBSD data. Moreover, the output results in TSL OIM ".ang" file format can be modified to be opened in commercial program for visual plotting without any difficulties. Figure 7(a) shows the packet map of the part 4, which was cut from grain 1 as mentioned above. The martensite variants are classified and colored by red, yellow, green and blue by parallel relationship between $(011)_{\alpha^{\prime}}$ and four variants of $(111)_{\gamma}$ close-packed plane (CP1 to CP4). Figure 7(b) represents sub-block (or variant) map of the same martensite region. The notation and orientation of 24 martensite variants from V1 to $\mathrm{V} 24$ were derived from eq. (1) using 24 conversion matrices $C_{i}$ given in Table 1 . The martensite variants are automatically indexed and highlighted by twenty four rainbow color codes from red to magenta. Low angle boundaries $\left(<15^{\circ}\right)$ between variants are shown in white color, while high angle boundaries $\left(\geq 15^{\circ}\right)$ are in black color. The obtained sub-block map (Fig. 7(b)) shows a typical morphology of low-carbon lath martensite, ${ }^{7,8)}$ in which thin laths of the same martensite variant grouped together to form sub-blocks. Pairs of sub-blocks with low angle boundary such as V1-V4, V2-V5 and V3-V6, which belong to the same packet, gathered together to form three types of block. The high angle boundaries between blocks or packets are sharp and clear. The low angle boundaries between sub-blocks are often unclear, since orientation of martensite gradually changed during switching from one martensite variant to another across the sub-block boundaries.

The misorientation of experimental points from the closest calculated martensite variants is also plotted in Fig. 7(c). It shows that the misorientation distribution has a local characteristic. The points with misorientation smaller than $3^{\circ}$ dominate with 0.84 fractions. The martensite areas with misorientation larger than $4^{\circ}$ occupy only 0.04 fractions. These higher misorientation areas are often found near high angle boundaries (block or packet boundaries) as well as near inclusion particles. ${ }^{9)}$ As mentioned in 4.2, the local high
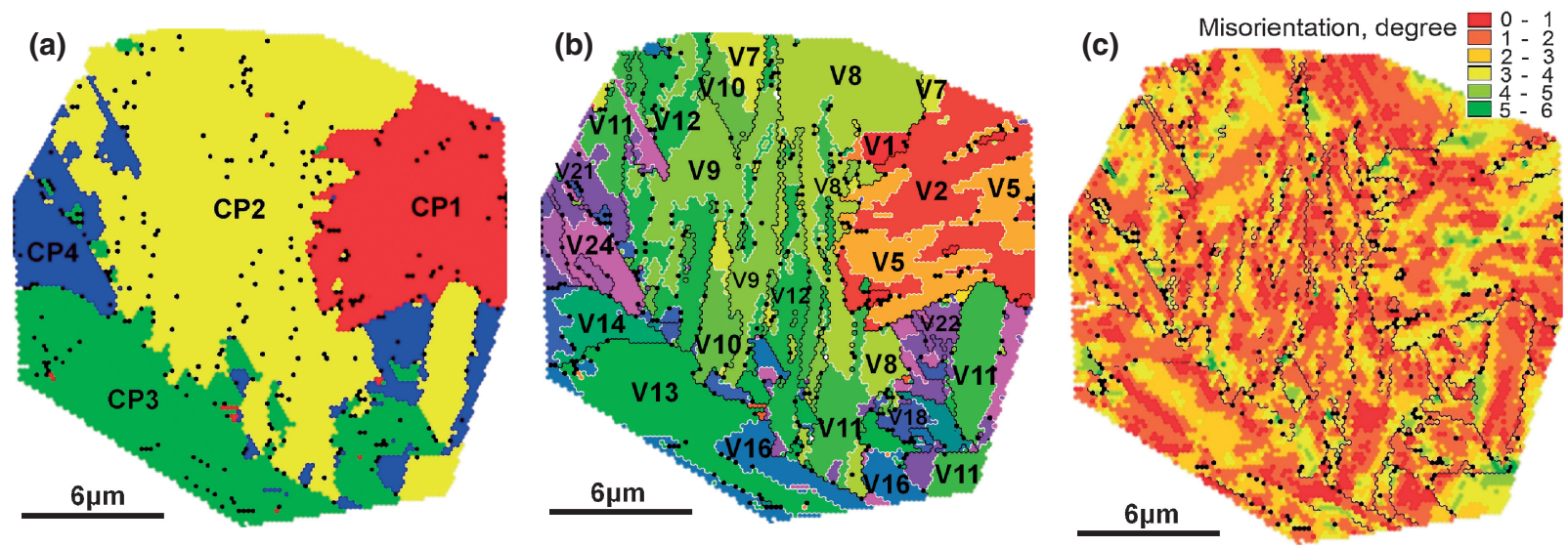

Fig. 7 Visual plotting of morphological analyses for part 4 of grain 1: (a) packet map showing packets of martensite colored by the same way as mentioned in Fig. 3; (b) sub-block map showing 24 colored variants of martensite grouped into sub-blocks (the black and white lines showing boundaries with angle $\geq 15^{\circ}$ and $<15^{\circ}$ respectively); (c) misorientation map showing misorientation of experimental points from the closest calculated martensite variants. 
misorientation of scan points from calculated martensite variants might be caused by local deformation of austenite due to transformation strain.

\section{Conclusions}

An advanced fitting method has been developed for crystallographic and morphological analyses of lath martensite basing on EBSD data. The main results can be summarized as below:

(1) The efficiency and precision of ordinary fitting method are improved by some advanced modifications of misorientation calculation and fitting procedure. The improved fitting method for EBSD data is capable for thorough crystallographic and morphological analyses of low-carbon lath martensite.

(2) Using coarse data with scanning step up to $1.6 \mu \mathrm{m}$ is an effective way to reduce calculation time without the risk of losing the precision. By this measure, a large number of prior austenite grains or a large grain can be effectively analyzed within reasonable time.

(3) When a sufficient part of a prior austenite grain was used for analyzed the tolerance of obtained orientation relationship is within $0.2^{\circ}$, while tolerance for fitting austenite orientation is within $0.5^{\circ}$.

(4) The obtained orientation relationship is almost constant for the given steel regardless the location on the sample of EBSD data set. The fitted orientation relationship is close to G-T type with $(111)_{\gamma}$ deviated $1.72^{\circ}$ from $(011)_{\alpha^{\prime}}$ and $[\overline{101}]_{\gamma}$ deviated $2.63^{\circ}$ from $[\overline{1} \overline{1} 1]_{\alpha^{\prime}}$. Austenite orientation is found to be locally changed within a prior austenite grain due to transformation strain, whereas the orientation relationship is almost unchanged.

\section{Acknowledgment}

This work was performed under the Inter-University Cooperative Research Program of the Institute for Materials Research, Tohoku University (Proposal No. 12K0063).

\section{REFERENCES}

1) G. V. Kurdjumov and G. Sachs: Z. Physik 64 (1930) 324-326.

2) S. Morito, H. Tanaka, R. Konishi, T. Furuhara and T. Maki: Acta Mater. 51 (2003) 1789-1799.

3) S. Morito, X. Huang, T. Furuhara, T. Maki and N. Hansen: Acta Mater. 54 (2006) 5323-5331.

4) G. Miyamoto, N. Takayama and T. Furuhara: Scr. Mater. 60 (2009) 1113-1116.

5) A. B. Greninger and A. R. Troiano: Metals Trans. 185 (1949) 590-598.

6) Y. He, S. Godet and J. J. Jonas: Appl. Cryst. 39 (2006) 72-81.

7) P. M. Kelly, A. Jostsons and R. G. Blake: Acta Metall. Mater. 38 (1990) 1075-1081.

8) V. Ocelík, I. Furár and J. Th. M. De Hosson: Acta Mater. 58 (2010) 6763-6772.

9) H. A. Pham, T. Ohba, S. Morito, T. Hayashi, T. Furuhara and G. Miyamoto: Mater. Sci. Forum 738-739 (2013) 25-30. 